Judit Borsy

\title{
Situation économique des orphelins du domaine du fonds public de Versend dans la première partie du $19^{\text {ème }}$ siècle
}

\begin{abstract}
The purpose of the study. The fundamental question is what factors influenced the living conditions of the 515 orphans left in the Versend estate between 1815 and 1848 . To what extent impacted the inherited wealth, the age of the orphaned child, the number of siblings, and the role of guardianship and lordship shaping the fate of orphans.

Applied methods. The orphan census and orphan documents of the Versend estate formed the basis of the research. With the help of data referring to their financial conditions, it was possible to compare the types of heritage and the handling of it. On the one hand, we examined the percentage distribution of all assets, and on the other hand, we performed calculations by filtering out different groups. The conclusions drawn from the figures were confirmed by examining individual examples. In the course of the research, in addition to our previous processing of the orphans of the Pécsvárad public foundation estate, we also reviewed the works related to the orphans in France.

Outcomes. Most of the orphans in Versend were very poor, and the loss of their parents made their situation much worse financially. The little more affluent only had the opportunity to learn, which mostly meant some kind of craftsmanship. Marriage also allowed orphans displaced from the family farm to get land, so orphans were married relatively early. Early deaths were affected by the scarcity of wealth, the number of siblings, the age of becoming an orphan. The fate of the orphans was basically determined by their financial situation, but its further development was influenced by the person of the guardian, their residence and circumstances, and even the solicitude of the orphan's guardian.
\end{abstract}

Keywords: Orphan, orphanage, Versend, manor, public foundation

L'importance de la famille et de son rôle protecteur est bien évident en examinant le sort des orphelins. Dans la première partie du $19^{\mathrm{meme}}$ siècle les pères et également les mères étaient menacés par la mort précoce. Les femmes ont abandonné beaucoup de fois leurs enfants très jeunes à cause de nombreuses accouchements. Les hommes se sont mariés à l'âge mûr, et c'est pourquoi ils ne pouvaient pas toujours élever leurs enfants.

Il ne faut pas expliquer à quel point les enfants ont souffert de l'absence de leurs parents, qu'ils ont essuyé une perte importante, mais il vaut la peine d'examiner les nombreux facteurs qui ont influencé leur situation économique.

Les parents qui sont restés seuls, dans la plupart des cas nétaient pas capables d'éduquer leurs enfants, surtout du point de vue financier. On était obligé de se remarier beaucoup de fois par nécessité matérielle. L'enfant ayant perdu son père, sa mère se trouvait dans la dépendence de son beau-père ou belle-mère.

Il arrivait, que les enfants nés de plusieurs mariages vivaient tous ensemble. Il n'est pas curieux si la veuve, la belle-mère des enfants du mariage premier ou second de son mari décédé, s'est remariée pour s'évader de cette situation. ${ }^{1}$

Dans ces familles „patchwork” il y avait beaucoup de problèmes si on n'avait fait ni un testament ni un contrat de mariage. On le voit dans l'exemple de Georges Klein, meunier de

1 On le voit dans l'exemple de la veuve de Jovan Knesovits qui a écrit dans sa plainte: „J'ai été forcée de me marier puisque mes beaux-fils ne m'avaient pas respectée.”MNL BaML XI.605. h.O100-102. Knezovits. 
Kéménd, père de deux filles qui avait acheté un moulin en 1803. Après sa mort, en 1810 sa veuve a épousé Jean Klósz. Il était aussi meunier de Kéménd et il a vendu son propre moulin pour pouvoir rénover le moulin vétuste de Georges Klein. Quand sa femme, la veuve de Georges Klein a été morte elle aussi, il s'est remarié à son tour avec Christine Loschan, et deux fils sont nés de leur mariage. Après la mort de Jean Klósz tous les orphelins voulaient acquérir le moulin, les filles puisque c'était leur père qui l'avait acheté et les garçons puisque c'était leur père qui l'avait rénové. ${ }^{2}$

La situation était encore plus compliquée où un veuf et une veuve se sont mariés ayant tous les deux des enfants nés d'un mariage précédent. La veuve de Nicolas Schleich avait deux enfants, mais elle soignait même les deux enfants nés du premier mariage de son mari décédé. Elle s'est remariée avec Martin Glück qui était aussi veuf et qui avait déjà, lui aussi, deux enfants nés de son premier mariage. Quatre enfants sont nés de leur mariage et quand les parents étaient morts, les dix héritiers ne savaient plus qui devait à qui et combien. ${ }^{3}$

En Hongrie dans la première partie du $19^{\text {ème }}$ siècle c'était le devoir du domaine de surveiller et d'administrer les biens des orphelins. ${ }^{4}$ En 1810 le conseil de lieutenance qui a dirigé les domaines du fonds public, ${ }^{5}$ a élaboré les instructions de l'administration de l'affaire des orphelins. D'après les instructions le domaine devait mettre en sécurité les biens des serfs, il devait surveiller le soignement des orphelins et décider en questions disputées. ${ }^{6}$

Dans la plupart des cas, l'héritage s'est divisé essentiellement en biens immeubles et en biens meubles, l'argent comptant ne s'y trouvait presque jamais. Les biens immeubles pouvaient être des vignes, des moulins, mais le plus souvent c'étaient des fonds de terre. ${ }^{7}$ En principe le serge nétait pas le possesseur du fonds de terre, mais en réalité ils pouvaient les vendre, ils pouvaient les changer entre les uns et les autres et ils pouvaient aussi en disposer par testament. Le seul critère en ait été de faire ratifier les contrats par le praefectus ${ }^{8}$, le chef du domaine seigneurial.

Vendre ou ne pas vendre les immeubles des orphelins c'était une question difficile, et c'était les chefs du domaine qui devaient en décider. Il y avait quatre solutions principales: 1.Vendre l'immeuble aux enchères 2 . le donner à louage (tipiquement les moulins, les vignes) 3. le tuteur (le beau-père ou la belle-mère) qui alimentait l'enfant s'en servait 4 . un des frères l'a gardé et il a payé la portion de l'héritage de ses frères et de ses soeurs.

La plupart des biens immeubles, 56,7\% étaient vendus aux enchères. ${ }^{9}$ Dans le $16,3 \%$ des cas c'étaient les veuves, dans le 5,8\% des cas c'étaient les tuteurs qui les ont gardés, et dans le 21,2\% des cas les biens immeubles sont restés dans la possession d'un des orphelins. Le provisor ${ }^{10}$ s'occupait de la vente des immeubles, mais les représentents de la municipalité (le juge et les

\footnotetext{
MNL BaML XI.605. h.O100-160. Klein, 161. Klósz. XI.605.i. Q 91/1821.

MNL BaML XI.605. h.O100-35. Schleich. 50. Glück.

4 Le tuteur des orphelins des paysans était le seigneur du domaine. Mezey, B. (2003): 114.

5 Le fonds public a été issu des biens des ordres religieux catholiques ayant abolis à la fin du $18^{\text {ème }}$ siècle. Le conseil de lieutenance a organisé l'administration de ces domaines. C'était en 1794 qu'on a partagé ces domaines d'après leur situation géographique en cinq districts, un desquels était celui de Pécsvárad. On a administré dans ce district sept domaines dont trois (celui de Pécsvárad, celui de Bozsok et celui de Versend) avaient fait partie de l'abbaye de Pécsvárad auparavant. Borsy, J. (2015): 183.

6 Borsy, J. (2018): 187-188.

7 En Hongrie c'était en 1767 que Marie Thérèse a réglé les relations entre le domaine seigneurial et les serges. Elle a précisé les prestations appartenues aux fonds de terre. Un terrain de serge s’est composé de terre arable (32 acres dans le comitat de Baranya) du jardin et du pré. Mais il y avait peu de paysans qui avait un "terrain entier", il y avait plutôt des terrains „demis”, „quarts” etc. Les paysans qui n’avaient qu'un huitième de terrain s'appelait „zsellér” (en latin: inquilinus) en Hongrie.

8 Praefectus: mot latin. Il était le chef du district du fonds public. Il était en relation du conseil de lieutenance et aussi des provisors des domaines. Borsy, J. (2007): 164-165.

9 Dans le domaine de Bozsok 40\% des vignes des orphelins nétaient pas vendus en 1813. Borsy, J. (2021): 58-59.

10 Provisor: mot latin. Il était le chef, l'intendant des domaines.
} 
assesseurs) étaient aussi présents aux enchères. Après avoir trouvé l'acheteur qui avait promis le plus, le traité de la vente devait être ratifié par le praefectus. Les ventes des enchères étaient accessibles à tous, on les a rendus public par des circulaires, en France par des affiches: „La vente des biens des mineurs doit être faite publiquement en présence du subrogé-tuteur, aux enchères qui seront reçues par un membre du tribunal de premier instance, ou par un notaire à ce commis, et à la suite de trois affiches apposées par trois dimanches consécutifs aux lieux accoutumés dans le canton."11

Les biens meubles ont été aussi vendus aux enchères. Il s'est produit que la veuve, ou l'un ou l'une des orphelins a présenté sa requête pour pouvoir garder quelque choses des biens meubles. C'étaient tipiquement le linge de maison, le linge de table, les vêtements (jupes, chemises, foulards etc.). Si on ne pouvait pas le prouver suffisamment, on devait les acheter aux enchères, si on avait de la chance à la mise à prix ou à prix modéré. En France c'était le tuteur qui devait s'occuper de vendre les biens des mineurs „... le tuteur est obligé de mettre son attention à vendre dans le mois, à partir de la cloture de l'inventaire, les meubles périssables, et les vendre..."12

Les membres de la famille et les créditeurs de la personne décédée étaient tous présents aux enchères, le succès desquelles était surtout leur intérêt. Dans les villages du domaine de Versend le peu des artisans et le manque des marchandises ont efforcé les habitants d'acheter même les articles usagés. La somme (venue du prix de la vente et l'argent comptant) a été mise dans la caisse pupillaire. Entre 1815 et 1840 on a gardé dans la caisse pupillaire du domaine du fonds public de Versend 225 biens (la plupart desquels ont été des capitaux) de 515 orphelins. ${ }^{13}$ (Tableau 1)

\section{Tableau 1: Nombre des capitaux et celui des orphelins dans les} villages du domaine de Versend (1815-1840)

\begin{tabular}{|l|l|l|}
\hline Nom du commun & Nombre des orphelins & Nombre des biens \\
\hline Versend & 72 & 35 \\
\hline Kéménd & 38 & 18 \\
\hline Szederkény & 15 & 5 \\
\hline Monyoród & 14 & 7 \\
\hline Maráza & 39 & 19 \\
\hline Liptód & 103 & 48 \\
\hline Kisnyárád & 20 & 7 \\
\hline Babarc & 107 & 46 \\
\hline Szajk & 107 & 40 \\
\hline Somme totale & 515 & 225 \\
\hline
\end{tabular}

Sources: MNL BaML XI.605.h-O 1-5. Árvaszámadási fókönyvek 1814-1840. 10-41. Árvaszámadások 1814-1848.

Le conseil de lieutenance a ordonné de donner à l'intèrêt l'argent des orphelains pour le fructifier. D'après l'explication de Dequiron: „Les capitaux des pupilles ne doivent pas rester oisifs entre les mains des tuteurs; la raison et la justice veulent qu'à l'exemple d'un bon père de famille ils les fassent fructifier." 14

\footnotetext{
11 Desquiron, A.T. (1810): 198
}

12 Desquiron, A.T. (1810): 181.

13 Le bien des orphelins (en latin: massa orphanorum, en hongrois: árva tömeg) signifiaient tout l'héritage, donc l'ensemble des biens immeubles (si on ne l'avait pas vendus), l'argent et même les lettres de crédit ou de dettes. Dans la caisse pupillaire on a gardé les capitaux des orphelins qui étaient enregistrés par les parents décédés même s'il y avait plusieurs héritiers qui se sont partagé l'héritage.

14 Desquiron, A.T. (1810): 186. 
Les intérêts servaient fondamentalement à agrandir le capital des orphelins pour qu'ils puissent le toucher en atteignant leur majorité. Il est intéressant que 10,1\% des orphelins majeurs ont laissé leur capital dans la caisse pupillaire probablement pour le faire fructifier plus longtemps.

Cette époque-là, en Hongrie on a atteint la majorité à l'âge de 24 ans, ou bien à l'état marié. ${ }^{15}$ En même temps en France l'âge de la majorité signifiait 21 ans, et on a strictement distingué la majorité de l'émancipation: „Le principal effet de l'emancipation est de mettre fin à la tutelle et rendre le mineur capable d'administrer ses biens. [...] l'emancipation n'est pas une pleine majorité. Le mineur émancipé pourra donc passer des baux dont la durée n'excédera pas neuf ans; il pourra recevoir ses revenus, en donner décharge, et faire tous les actes qui sont de pure administration, sans être restituable contre ces actes dans tous les cas où le majeur ne le serait pas lui même; mais il ne pourra point affecter le fonds qui constitue son patrimoine. Il ne pourra intenter une action immobiliaire, ni défendre, même recevoir et donner décharge d'un capital mobilier sans l'assistance de son curateur qui au dernier cas surveillera l'emploi du capital reçu." 16

En Hongrie cette distinction n'existait pas, l'orphelin qui s'était marié pouvait recevoir son héritage. Quand il s'agissait d'une orpheline, elle l'a touché en compagnie de son mari qui devait lui aussi signer pour acquit.

Avant la majorité on ne pouvait toucher que les intérêts pour des dépenses irrégulières. Par exemple Jean Handler aprenti tailleur de Babarc a demandé les intérêts de son capital pour pouvoir acheter les matériaux nécessaires pour son ouvrage de maître. ${ }^{17}$ Autre exemple quand Catherine Stein orpheline de 17 ans avait besoin de l'argent pour se préparer à son mariage. Dans ces cas il fallait toujours demander la permission du praefectus du district. ${ }^{18}$

Les intérêts pouvaient servir aussi à l'éducation des orphelins. Il arrivait beaucoup de fois que le tuteur a demandé régulièrement de l'argent pour l'alimentation de l'orphelin. C'était le tribunal seigneuria ${ }^{19}$ qui avait le droit de donner la permission et en général on pouvait recevoir une somme fixée jusqu'à l'âge de 12 ans de l'orphelin. Dans ces cas bien entendu, le capital de l'orphelin ne s'est point élevé. ${ }^{20}$

Il est évident donc, que la personne du tuteur était très important même du point de vue de la situation matérielle de l'orphelin. La question, la plus importante était s'il avait dépensé les intérêts du capital de l'orphelin ou non. L'orphelin ayant été sous la tutelle de celui qui n'avait pas touché les intérêts pour la nourriture, pour les vêtements, atteignant sa majorité a reçu une somme plus signifiant que l'enfant soigné par celui qui avait dépensé tous les intérêts du capital.

D'après la distinction de Zsoldos, expliquée dans son oeuvre, le tuteur surveille la personne, le curateur les biens. ${ }^{21}$ Le domaine était donc le curateur, mais le tuteur de l'orphelin nétait pas toujours son soigneur réel, le tuteur et le soigneur de l'orphelin pouvait être des personnes différentes. Si par exemple la mère était en vie elle a soigné son enfant, mais le tuteur était toujours un homme, un oncle, le beau-père, un parent etc. C'est à cause de cela que la proportion des mères parmi les tuteurs est si petite. (Tableau 2)

Zsoldos, I. (1842): 45.

Desquiron, A.T. (1810): 256-257.

MNL BaML XI.605. h.O100-148. Handler.

MNL BaML XI.605. h.O100-137. Stein.

19 Sedis dominalis en latin, úriszék en hongrois. Le tribunal du domaine où on a réglé les affaires des paysans, des serfs.

20 Borsy, J. (2019): 351.

21 Zsoldos, I. (1842): 45. 
Tableau 2: La personne du tuteur

\begin{tabular}{|l|l|l|}
\hline Tuteur & Nombre & Pour cent \\
\hline Père ou mère & 13 & 2,5 \\
\hline Grands-parents & 4 & 0,8 \\
\hline Frère ainé & 48 & 9,3 \\
\hline Beau-frère (mari de la soeur) & 16 & 3,1 \\
\hline Cousin & 3 & 0,5 \\
\hline Oncle & 41 & 8,0 \\
\hline Parrain & 113 & 22,0 \\
\hline Beau-père (mari de la mère) & 74 & 14,4 \\
\hline Étranger (notaire, curé, instituteur) & 53 & 10,3 \\
\hline Orphelin majeur ou marié & 52 & 10,1 \\
\hline Manque d'information & 98 & 19,0 \\
\hline Somme totale & 515 & 100,0 \\
\hline
\end{tabular}

Sources: MNL BaML XI.605.h-O 1-5. Árvaszámadási fôkönyvek 1814-1840. 100. Árvák iratai.

En examinant les tuteurs des orphelains du domaine de Versend on voit que le „parent choisi" le parrain jouait souvent un rôle important dans la vie de l'orphelin à ce temps-là, 22\% des tuteurs étaient des parrains. Il arrivait souvent que le frère ainé ou la soeur ainée étant déjà mariée s'est chargé de son frère cadet ou de sa soeur cadette. $12,4 \%$ des tuteurs étaient le frère ou la soeur pour mieux dire le mari de celle ci. Le beau-père était le tuteur encore plus souvent, $14,4 \%$ des cas, ce qui prouve les remariages fréquents des veuves.

La personne du tuteur a influencé même le domicile de l'orphelin. C'était plus que la moitié des orphelins qui étaient soignés par un tuteur ayant un domicile lointin. Il nétait pas facile de trouver quelqu'un dans le même village qui pouvait se charger d'un orphelin, mais ce qui était presque impossible c'était de trouver un tuteur qui voulait se charger de plusieurs orphelins. Lorphelin en dehors de la perte de son père et de sa mère, il a perdu même son domicile d'origine et qui était encore pire, il devait se séparer ses frères et ses soeurs comme c'était souvent le cas. En 1830 les trois enfants Mikli d'origine Szajk restés sans parents à l' âge de 3, 5 et 6 ans, étaient soignés par leur grand-père et ils habitaient chez lui à Versend. Après sa mort on a séparé le cadet de deux ainés et leurs domiciles et leurs tuteurs se sont changés quatre fois dans leur enfance. ${ }^{22}$

Dans le domaine de Versend c'était un peu plus que le tiers des orphelins qui sont restés à leur domicile d'origine. 105 orphelins sont restés dans un des villages du domaine de Versend, 117 orphelins n”ont pas quitté le comitat de Baranya, et il y avait 26 orphelins qui ont déménagé dans un autre comitat (Tolna, Verőce, Moson, Fejér, Temes, Veszprém), 13 sont devenus soldat, 56 sont décédés. (Tableau 3)

22 MNL BaML XI.605. h.O100-166. Mikli. 
Tableau 3: Domiciles des orphelins et des orphelines après la mort de leurs parents

\begin{tabular}{|l|l|l|l|l|l|l|}
\hline Domicile & Orphelin & $\begin{array}{l}\text { Pour } \\
\text { cent }\end{array}$ & Orpheline & $\begin{array}{l}\text { Pour } \\
\text { cent }\end{array}$ & $\begin{array}{l}\text { Tous les } \\
\text { orphelins }\end{array}$ & $\begin{array}{l}\text { Pour } \\
\text { cent }\end{array}$ \\
\hline Reste á son domicile d'origine & 107 & 40,5 & 91 & 36,2 & 198 & 38,4 \\
\hline $\begin{array}{l}\text { Reste dans le domaine } \\
\text { de Versend }\end{array}$ & 45 & 17,1 & 60 & 23,9 & 105 & 20,4 \\
\hline $\begin{array}{l}\text { Habite dans un village } \\
\text { du comitat Baranya }\end{array}$ & 51 & 19,3 & 66 & 26,3 & 117 & 22,7 \\
\hline Habite dans un autre comitat & 16 & 6,1 & 10 & 4,0 & 26 & 5,1 \\
\hline Soldat & 13 & 4,9 & - & - & 13 & 2,5 \\
\hline Décédé & 32 & 12,1 & 24 & 9,6 & 56 & 10,9 \\
\hline Somme totale & 264 & 100,0 & 251 & 100,0 & 515 & 100,0 \\
\hline
\end{tabular}

Sources: MNL BaML XI.605.h-O 1-5. Árvaszámadási főkönyvek 1814-1840. 100. Árvák iratai.

En mettant les filles en comparaison des garçons on voit qu'il y avait moins de filles qui n’ont pas quitté leur domicile d'origine, mais il y a plus de garçons qui ont déménagé à un lieu plus lointain.

Bien que la grandeur des capitaux des orphelins gardés dans la caisse pupillaire ne reflète pas toujours leur situation matérielle réelle, il peut être quand même intéressant à examiner le changements des nombres et des sommes de ces capitaux. (Tableau 4)

Tableau 4: Comparaison de la grandeur et le nombre des capitaux en 1815 et en 1840 (en forint ${ }^{23}$ )

\begin{tabular}{|l|l|l|l|l|l|l|l|l|}
\hline $\begin{array}{l}\text { grandeur } \\
\text { des biens }\end{array}$ & $\begin{array}{l}\text { nombre } \\
\text { des } \\
\text { capitaux } \\
\text { en 1815 }\end{array}$ & $\%$ & $\begin{array}{l}\text { nombre } \\
\text { des } \\
\text { capitaux } \\
\text { en } 1840\end{array}$ & $\%$ & $\begin{array}{l}\text { somme } \\
\text { des } \\
\text { capitaux } \\
\text { en 1815 } \\
\text { en forint }\end{array}$ & $\%$ & $\begin{array}{l}\text { somme } \\
\text { des } \\
\text { capitaux } \\
\text { en 1840 } \\
\text { en forint }\end{array}$ & $\%$ \\
\hline $0-100$ & 33 & 34,7 & 19 & 27,5 & 1547 & 5,1 & 981 & 1,8 \\
\hline $101-200$ & 19 & 20,0 & 13 & 18,8 & 2525 & 8,2 & 1945 & 3,7 \\
\hline $201-300$ & 16 & 16,9 & 7 & 10,2 & 4290 & 14,0 & 1753 & 3,3 \\
\hline $301-500$ & 10 & 10,5 & 9 & 13,0 & 3789 & 12,4 & 3257 & 6,1 \\
\hline $501-1000$ & 11 & 11,6 & 7 & 10,2 & 7238 & 23,7 & 4856 & 9,1 \\
\hline $1001-1500$ & 2 & 2,1 & 6 & 8,7 & 2197 & 7,2 & 7059 & 13,3 \\
\hline plus de 1501 & 4 & 4,2 & 8 & 11,6 & 8979 & 29,4 & 33347 & 62,7 \\
\hline Somme totale & 95 & 100 & 69 & 100 & 30565 & 100 & 53198 & 100 \\
\hline
\end{tabular}

Sources: MNL BaML XI.605.h-O 1-5. Árvaszámadási fôkönyvek 1814-1840. 100. Árvák iratai.

En mettant la grandeur des capitaux de l'année 1815 en comparaison avec celle de l'année 1840 on voit bien que le nombre des petites fortunes se sont réduites presqu'à moitié pendant les 25 ans. En 1815 le nombre des capitaux moins de 300 forints était 71,6\% du nombre total des capitaux, tandis qu'en 1840 ce n'était que 56,5\%. Le nombre des capitaux plus de mille forints en 1815 était 6,3\% du nombre total et en 1840 c'était 20,3\%. En même temps la proportion de la somme des capitaux moins de 300 forints s'est diminuée de $27,3 \%$ à $8,8 \%$. Par contre la proportion de la somme des capitaux plus de mille forints a augmenté de 36,6\% à 76\%.

23 Forint, florin en latin florenus, devise de la Hongrie de 1750 à 1892. 
Pendant les 25 ans le nombre des capitaux s'est diminué à 72,6\%, tandis que la somme des capitaux s'est élevé à $172,0 \%$. Il est facile de voir que les tout petits capitaux nétaient plus gardés dans la caisse pupillaire, et que la somme des capitaux a augmenté beaucoup.

On voit la même chose si on regarde tous les 225 capitaux qu'on a gardés dans la caisse pupillaire de Versend entre 1815 et 1847 . Parmi les capitaux des orphelins il y avait 46 qui n'atteignaient pas 100 forints et 103 qui étaient au-dessous de 500 forints. Il n'y avait que 11 capitaux au-dessus de 2000 forints. (Tableau 5)

Tableau 5: La grandeur et le nombre de 225 capitaux entre 1815 et 1847

\begin{tabular}{|l|l|l|}
\hline la grandeur des capitaux & nombre des capitaux & $\%$ \\
\hline manque de l'argent & 9 & 4,0 \\
\hline $1-100$ & 46 & 20,4 \\
\hline $101-500$ & 103 & 45,8 \\
\hline $501-1000$ & 33 & 14,7 \\
\hline $1001-1500$ & 16 & 7,1 \\
\hline $1501-2000$ & 7 & 3,1 \\
\hline au-dessus de 2000 & 11 & 4,9 \\
\hline Somme totale & 225 & 100 \\
\hline
\end{tabular}

Sources: MNL BaML XI.605.h-O 1-5. Árvaszámadási főkönyvek 1814-1840. 100. Árvák iratai.

En examinant la grandeur des capitaux la question la plus importante: combien d'orphelins se sont partagé l'héritage. Dans le tiers des cas l'héritage appartenait à un seul orphelin, dans la moitié des cas l'héritage appartenait à deux ou à trois orphelins et dans les cas qui restent $(16,4$ pour cents) l'héritage a été partagé entre quatre ou plus de quatre orphelins. D'un autre point de vue ce n'est que $15,5 \%$ des orphelins qui a hérité seul, la moitié des orphelins avaient un ou deux frères et soeurs, et le tiers des orphelins en avaient plus de trois. (Tableau 6)

Tableau 6: Le nombre des orphelins et des capitaux de la caisse pupillaire

\begin{tabular}{|l|l|l|l|l|l|l|l|l|}
\hline & $\begin{array}{l}1 \\
\text { orphelin }\end{array}$ & $\begin{array}{l}2 \\
\text { orphelins }\end{array}$ & $\begin{array}{l}3 \\
\text { orphelins }\end{array}$ & $\begin{array}{l}4 \\
\text { orphelins }\end{array}$ & $\begin{array}{l}5 \\
\text { orphelins }\end{array}$ & $\begin{array}{l}6 \\
\text { orphelins }\end{array}$ & $\begin{array}{l}7 \\
\text { orphelins }\end{array}$ & $\begin{array}{l}\text { Somme } \\
\text { totale }\end{array}$ \\
\hline $\begin{array}{l}\text { nombre } \\
\text { des biens }\end{array}$ & 80 & 61 & 47 & 20 & 11 & 5 & 1 & 225 \\
\hline$\%$ & 35,6 & 27,1 & 20,9 & 8,9 & 4,9 & 2,2 & 0,4 & 100 \\
\hline $\begin{array}{l}\text { nombre } \\
\text { des } \\
\text { orphelins }\end{array}$ & 80 & 122 & 141 & 80 & 55 & 30 & 7 & 515 \\
\hline pour cent & 15,5 & 23,7 & 27,4 & 15,5 & 10,7 & 5,8 & 1,4 & 100 \\
\hline
\end{tabular}

Sources: MNL BaML XI.605.h-O 1-5. Árvaszámadási főkönyvek 1814-1840. 100. Árvák iratai.

Il est évident que les petits capitaux gardés dans la caisse pupillaire n'ont pu donné que peu d'intérêts, et comme ça il n'était assez ni pour l'éducation de l'orphelin ni pour agrandir sa fortune. Mais dans les cas où l'orphelin avait très peu d'argent ou il n'en avait pas du tout, quelques fois il était le possesseur d'un biens immeuble, d'un vigne, d'un moulin ou d'un fonds de terre.

Le traitement de ces biens immeubles était différent. Les terrains étaient cultivés le plus souvent par la belle-mère, par le beau-frère (le mari de la soeur ainée), ou par le tuteur. Les moulins et les vignes étaient beaucoup de fois en location, le prix de laquelle servait à l'éducation de l'enfant. Parfois quelqu'un s'est chargé de cultiver le vigne en échange de la récolte. Il est vrai que dans ces cas l'orphelin n'a pas reçu d'intérêts régulièrement, à l'aide duquel on aurait pu 
résoudre ses problèmes financiers mais si c'était l'orphelin qui était resté le possesseur du bien immeuble, atteignant la majorité, il pouvait gagner de quoi vivre. Hélas, il est arrivé aussi que le locataire n'a pas voulu redonner à l'orphelin majeur le biens, c'étaient les cas où le tribunal seigneurial a pris une décision.

La grande partie des parents décédés des orphelins du domaine de Versend étaient des cultivateurs ayant des fonds de terre, la grandeur desquels étaient très différente. Parmi les 225 pères il n'y avait que sept meuniers, cinq employés du domaine (forestier, chasseur, tonnelier, haïdouk), un aubergiste, et deux artisans qui vivaient probablement à un niveau un peu plus élevé.

En examinant le sort des orphelins on voit qu'il n'y avait que peu qui ait pu continuer des études. Parmi les 264 garçons il ny avait que 43 qui savaient sûrement souscrire leur nom, parmi les 251 filles il n'y en avait que trois. Les filles n'ont pas fait des études même si elles avaient une fortune considérable. Madelaine Helcher, qui avait le plus grand capital parmi les orphelins du domaine de Versend, s'est mariée à l'âge de 14 ans. Elle a pu toucher son héritage de 6429 forints augmenté par les intérêts à 8900 forints. ${ }^{24}$ Madelaine Klein qui était aussi fortunée ayant un capital de 5836 forints, nont suivi que des cours de broderie à Pécsvárad. Elle a épousé un meunier fortuné. ${ }^{25}$

Bien qu'on n'ait pas d'informations de ceux qui étaient encore trop jeunes en 1847 pour pouvoir choisir un métier, il vaut la peine d'examiner la relation entre les professions choisies par les garçons et les capitaux gardés dans la caisse pupillaire. (Tableau 7)

Tableau 7: Professions des 264 orphelins

\begin{tabular}{|l|l|l|l|l|}
\hline Professions des orphelins & $\begin{array}{l}\text { le nombre des } \\
\text { orphelins }\end{array}$ & $\%$ & $\begin{array}{l}\text { somme du } \\
\text { capital }\end{array}$ & $\begin{array}{l}\text { en } \\
\text { moyenne }\end{array}$ \\
\hline Maître d'école & 3 & 1,1 & 5542 & 1847 \\
\hline Aubergiste & 1 & 0,4 & 1727 & 1727 \\
\hline Meunier & 5 & 1,9 & 6072 & 1215 \\
\hline Artisan & 34 & 12,9 & 15566 & 458 \\
\hline Cultivateur & 11 & 4,2 & 2998 & 272 \\
\hline Décédé & 56 & 21,2 & 11319 & 202 \\
\hline Valet & 5 & 1,9 & 711 & 143 \\
\hline „Zsellér” & 1 & 0,4 & 140 & 140 \\
\hline Soldat & 17 & 6,4 & 1902 & 112 \\
\hline Manque d'information & 131 & 49,6 & - & - \\
\hline Somme totale & 264 & 100 & 45977 & 345,7 \\
\hline
\end{tabular}

Sources: MNL BaML XI.605.h-O 1-5. Árvaszámadási főkönyvek 1814-1840. 100. Árvák iratai.

Ceux qui avaient un capital au dessous de 200 forints sont devenus beaucoup de fois des soldats, des serviteurs. Parmi les 86 orphelins qui avaient les plus petits capitaux il y avait 6 qui sont devenus soldats, et deux qui ont réussi de devenir à un propriétaire terrien en se mariant.

Apprendre quelque métier nétait pas facile surtout du point de vue financier. Les capitaux de ceux qui sont devenus des artisans étaient en moyenne 458 forints, mais on trouve cinq sommes au-dessus de mille forints, dont le plus grande est 2696 forints. Presque la moitié des métiers choisis avait le rapport de la préparation des vêtements: tailleur, cordonnier, tisserand, pelletier, chapelier, tisseur de bas.

24 MNL BaML XI.605. h.O100-190. Helcher.

25 MNL BaML XI.605. h.O100-160. Klein. 
Les orphelins qui sont devenus meuniers avaient les capitaux les plus grands, comme on voit dans l'exemple de Valentin Hellebrand. Il est devenu orphelin à lâge de 6 ans. Il avait eu un frère aîné qui était mort à l'âge de 9 ans, comme ça Valentin est resté le seul héritier d'un capital de 4751 forints. Son oncle était le tuteur, qui n'avait touché que 20 forints par an pour l'éducation de son neveu. Il a acheté des manuels $\mathrm{ABC}$, tableau à calculer etc. Á l'âge de vingt ans il était déjà meunier, et il a touché son capital qui avait augmenté avec les intérêts à 6386 forints, 134\% de son héritage original. ${ }^{26}$

Devenir à un maitre d'école était presqu'un choisi de luxe. Il fallait acheter des manuels, des vêtements et parfois des instruments de musique. Georges Lutz avait un capital de 3700 forints dans la caisse pupillaire. Son tuteur a touché 300 forints par an à titre de l'éducation de l'orphelin. Il a dépensé beaucoup, il a acheté même un violon et un piano pour l'orphelin. Il tenait nécessaire que l'orphelin puisse jouer de l'instrument de musique même le soir et le matin dans sa chambre. Georges Lutz est devenu instituteur, mais son capital restait 3700 forints, puisque les intérêts étaient tous dépensés pendant les années. ${ }^{27}$

Pour pouvoir toucher le capital les orphelins devaient le demander du praefectus. Le domaine n'a pas donné volontiers le capital à un orphelin solitaire. Même si on a déjà atteint la majorité, on a accentué son état marié. Par exemple quand Thérese Travers a demandé son capital de 500 forints on lui a répondu qu'il valait mieux de garder son argent dans la caisse jusqu'à ce qu'elle ne se marie pas. ${ }^{28}$ Il n'y a qu'une exemple exceptionnelle où une fille à l'âge de 20 ans ait reçu son capital. Thérese Strumpf domiciliée à Vienne, avait une profession et comme ça elle a reçu la permission de toucher son argent bien quelle n'ait pas été mariée. ${ }^{29}$

Quelques orphelins ont pu acquérir le fonds de terre de la famille. C'était une bonne solution pour celui qui avait pu payer la part successorale à ses frères et à ses soeurs et comme ça il est resté le seul possesseur de l'héritage. Il arrivait bien fois que le frère ainé s'est chargé de la tuterelle et le soignement de ses petits frères, comme c'était le cas de Jean Raith de Szajk. Les parents ont été morts en 1821, et le fils ainé avait acquis le fonds familial tandis que ses frères cadets nen avaient rien reçu. Il est vraie qu'il devait élevé ses deux frères et trois soeurs pendant des années. ${ }^{30}$ Lun de ses frères, Jacques Raith avait de la chance, en 1830 il est devenu le possesseur d'un fonds de terre assez grand par son mariage avec Elisabeth Müller. ${ }^{31}$

Le mariage signifiait une nouvelle famille pour les filles mais même pour les garçons. En dehors du point de vue sentimental il y avait même le point de vue matériel. Les pères n'ayant pas de fils ont fait souvent un contrat de mariage avec leur gendre en lui donnant tout son biens, le fonds de terre, les vignes, la maison. Par ce contrat de mariage l'orphelin est devenu immédiatement le possesseur du bien.

Jean Schleicher de Kéménd a épousé Elisabeth Stürzenberger de Szajk en 1830, d'après leur contrat de mariage son beau-père lui a donné sa propriété foncière, son vigne et le pressoir avec tous ses accessoires. ${ }^{32}$

Il y avait 288 orphelins qui se sont mariés, 41 étaient déjà marié quand leurs parents étaient morts. L'âge moyen des filles au moment du mariage était 20 ans et 6 mois, celui des garçons était 22 ans 8 mois. Mais il est intéressant que ceux qui se sont mariés dans les premiers trois ans après la mort de leurs parents étaient en moyenne plus jeunes, les filles avaient 18 ans 6 mois les garçons avaient 20 ans 10 mois. Il semble que ceux qui étaient devenus orphelins à l'âge nubile

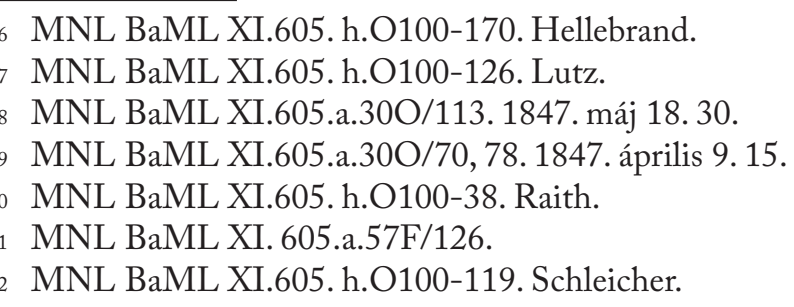


ont fait tous leurs efforts pour se marier le plus tôt, et trouver comme ça une nouvelle famille qui leur offrait la sécurité sentimentale, et matérielle.

En examinant les cas mortels il y avait 56 orphelins (10,9\%) qui étaient morts très jeunes. L'âge des orphelins décédés était tout différent, mais c'était à l'âge de 12 à 15 ans que la plupart de ces enfants ont perdu leurs parents. Peut être que les enfants très petits ont été entretenus soigneusement, les jeunes plus agés pouvaient déjá se soigner eux- mêmes, mais les enfants qui avaient plus de douze ans n'ont pas reçu un traitement convenable.

Les enfants décédés avaient de petites fortunes, 202 forints en moyenne. Il est évident que ces petites sommes n'avaient pas d'intérêts signifiants, mais en plus le domaine n'ont pas permis les toucher pour l'éducation des enfants âgés plus de 12 ans, puisqu'ils pouvaient déjà travailler. Ils étaient donc obligés de travailler le plus souvent comme valet, domestique. Parmi les 56 orphelins il n'y avait que 3 qui n'avaient pas de frères ou soeurs. On peut supposer que les enfants qui s'étaient habitués à une famille de nombreux enfants, en perdant leurs parents, en se séparant de leurs frères ou soeurs étaient devenus encore plus vulnérables que ceux qui n'avaient eu jamais de frères ou soeur.

Le sort des orphelins du domaine de Versend a dépendu de plusieurs facteurs. Il est presque évident que le bien héritié a défini leur état de fortune, mais le domaine a joué aussi un rôle important. Jean Kiss était le provisor de Versend de 1808 à 1835. Il était un homme ponctuel qui avait surveillé les biens des orphelins. En examinant 33 procès verbaux des ventes aux enchères entre 1814 et 1830 à Versend on voit que le prix de vente a surpassé la mise à prix par 38,6\% en moyenne, ce qui a augmenté le capital des orphelins. Donner à l'intérêt l'argent c'était le devoir du provisor. Dans le domaine de Versend on a prêté presque toujours les 90-95\% de l'argent des orphelins. Il était important encore la personne du tuteur qui a influencé le domicile, et la profession choisie des orphelins. Mais en regardant le sort des 515 orphelins de Versend, on peut dire que les orphelins avaient assez peu de possibilités d'atteindre le niveau de vie, la situation de fortune de leurs parents.

\section{Bibliographie}

Borsy, J. (2007), A Pécsváradi közalapitványi kerület tisztviselói, alkalmazottai a 19. század elsó felében, in: Nagy Imre Gábor (éd.), Baranyai Történelmi Közlemények 2. Pécs: BML, 161-221.

Borsy,J. (2015), A pécsváradi közalapitványi uradalom gazdálkodásának sajátosságai az 1830-as években, in: Szirácsik Éva (éd.), Régi nagybirtokok, új kutatások. Dominium I. Budapest: Unicus Múhely, 183-201.

Borsy, J. (2018), Gondoskodás az árvákról a pécsváradi közalapitványi kerület baranyai uradalmaiban a 19. század elsö negyedében, in: Szirácsik Éva (éd.), A földesurak szerepe. Dominium IV. Budapest: Unicus Múhely, 186-210.

Borsy,J. (2019), A pécsváradi közalapitványi uradalmak árvaügyi iratainak kutatási lehetóségei és nehézségei. in: Lengvári István - Pilkhoffer Mónika - Vonyó József (éd.), Az ember helye - a hely embere. BudapestPécs, 344-358.

Borsy, J.: (2021), Der Weinbau der Leibeigenen in den Herrschaften des Pécsvárader Stiftungsdistrikts in der ersten Hälfte des 19. Jabrbunderts. in: Zoltán Kaposi, Virág Rab, (éd.) Economic and Social Changes: Historical Facts: Analyses and Interpretations. Working Pécs, 53-60.

Desquiron, A.T. (1810), Traité de la minorité, de la tutelle et de l'émancipation, Paris: D.Colas, ImprimeurLibraire.

Mezey, B. (2003), Magyar jogtörténet, Budapest.

Zsoldos, I. (1842), Szolgabirói bivatal. Közrendtartási rész. Pápa. 


\section{Sources}

MNL BaML = Magyar Nemzeti Levéltár Baranya Megyei Levéltára

XI. 605. Pécsváradi közalapítványi kerület levéltára

XI. 605. a. Főtiszti iratok

XI. 605. h. Versendi uradalom iratai 